\title{
FINAL REPORT FOR
}

\section{Integrated Experimental and Theoretical Study of the \\ Thermophysical Properties of Fluid Mixtures:}

\section{PROPERTIES OF MIXTURES NEAR A PHASE TRANSITION}

February 1, 1992 - January 31, 1996

Prepared for

\author{
Dr. F. D. Stevenson \\ Division of Chemical Sciences \\ Office of Basic Energy Sciences \\ U.S. Department of Energy \\ Washington, DC 20585
}

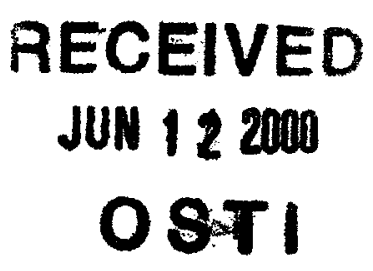

by

Physical and Chemical Properties Division Chemical Science and Technology Laboratory National Institute of Standards and Technology

Boulder, CO 80303-3328

$\begin{array}{llll}\text { Principal Investigators } & \text { H.J.M. Hanley } & \begin{array}{l}\text { (303) } 497-3320 \\ \text { D.G. Friend }\end{array} & \begin{array}{l}\text { (303) } 497-5424 \\ \text { howard.hanley@nist.gov } \\ \text { daniel.friend@nist.gov }\end{array} \\ \text { Admision Chief, Acting } & \text { R. Huie } & \text { (301) } 975-2559 & \text { robert.huie@nist.gov } \\ \text { Telefacsimile Number } & & \text { (303) } 497-3868 & \text { beverly.armstrong@nist.gov } \\ & & \text { (303) } 497-5224 & \end{array}$

November, 1999 


\subsection{Introduction}

In this report we highlight the progress and significant accomplishments made during the period February 1, 1992 through January 31, 1996 on DoE Grant DE AI05-89ER13992. Because a final progress report was prepared and forwarded to the Department of Energy Division of Chemical Sciences in July 1994, we emphasize descriptions of work completed in the final year and one-half of the project. The primary goal of the work is to study fundamental problems in the area of fluid thermophysical properties in order to develop predictive models capable of calculating fluid properties and phase boundaries for a variety of pure and mixture systems of importance to the energy and chemical industries. Our efforts are divided among three general areas:

1) Scattering studies to investigate the microscopic structure of real and model liquid systems.

2) Computer simulation, theory of the solid-liquid phase boundary, and theory of kinetic processes in fluids.

3) Properties near the critical locus emphasizing mixture phase equilibrium near liquid-vapor and liquid-liquid criticality, as well as dynamical processes in near-critical mixtures.

Significant results have been achieved in each of these areas during the performance of this contract. In the next section of this report, we give very brief descriptions of our activities and findings; these are generally grouped according to the general areas given above, although there is considerable overlap among these activities. Short narrative discussions of selected research areas are given in Section 3. We have attempted to keep this report short; more extensive discussions of our activities are available in the cited references. If any additional information is needed, please contact the principal investigators or an author of any of the publications. Lists of related publications and presentations are given in the last section of this report.

\subsection{Summary of Research Findings}

2.1 We have succeeded in examining the structure of model liquid mixtures by neutron scattering in contrast-matched $\mathrm{D}_{2} \mathrm{O} / \mathrm{H}_{2} \mathrm{O}$ solvents. These studies involved mixtures of polystyrene and silica suspensions which were imaged at the Cold Neutron Research Facility at NIST.

2.2 We have been able to estimate partial structure factors in mixtures from scattered neutron intensities, although multiple scattering and the determination of particle form factors have presented formidable obstacles.

2.3 We have successfully applied a density functional approach to the freezing transition of a colloidal system; the neutron studies indicate that a polycrystalline form seems to dominate.

2.4 In dilute aqueous suspensions of protonated and deuterated polystyrene (which form model atomic fluid mixtures), we have examined structures in both equilibrium and sheared systems. The equilibrium results conform with our understanding of microstructures, although the explicit 


\section{DISCLAIMER}

This report was prepared as an account of work sponsored by an agency of the United States Government. Neither the United States Government nor any agency thereof, nor any of their employees, make any warranty, express or implied, or assumes any legal liability or responsibility for the accuracy, completeness, or usefulness of any information, apparatus, product, or process disclosed, or represents that its use would not infringe privately owned rights. Reference herein to any specific commercial product, process, or service by trade name, trademark, manufacturer, or otherwise does not necessarily constitute or imply its endorsement, recommendation, or favoring by the United States Government or any agency thereof. The views and opinions of authors expressed herein do not necessarily state or reflect those of the United States Government or any agency thereof. 


\section{DISCLAIMER}

Portions of this document may be illegible in electronic image products. Images are produced from the best available original document. 
dependence on number fraction needs clarification; the distortion of the structure when a shear is applied is clearly seen in this technique, and we learn how the distortion affects the system's properties.

2.5 Our neutron scattering study of the microstructure of conventional fluid mixtures focussed on the cyclohexane + duodecane system with differential scattering from ${ }^{12} \mathrm{C}$ and ${ }^{13} \mathrm{C}$ and ${ }^{1} \mathrm{H}$ and ${ }^{2} \mathrm{H}$ (D) suostitutions. The results from the Los Alamos Neutron Scattering Center were encouraging.

2.6 In a small angle neutron scattering (SANS) investigation of a suspension of sodium montmorillonite, we were able to observe the change in scattered intensity when a shear is imposed. With an assumption of disc-like morphology, we were able to show that, at high Peclet numbers, the normal vector of the disk is preferentially oriented parallel to the imposed velocity gradient.

2.7 In our SANS and light scattering studies of reversible reactions in micellar systems, we have learned, that under shear, small spherical micelles can grow to form worm-like macromolecules which, unlike conventional polymers, have dynamics that are clearly shear dependent.

2.8 We have clarified the relationship between the rheological behavior of a dense colloidal suspension and changes in its microstructure by coupling rheological studies with small angle neutron scattering.

2.9 Our SANS studies of aqueous silica gels show the variation of gel structure with volume fraction and indicate an effect of applied shear on the final gel structure.

2.10 The problem of aggregation of clay platelet dispersions is being addressed by the formation of platelet-surfactant micelles which are being studied using $\mathrm{x}$-ray diffraction, SANS, and electron microscopy.

2.11 We have introduced a novel thermostat in our molecular dynamics simulations which makes no assumptions about the form of the streaming velocity; this has enabled us to study simple liquids under very high Reynolds numbers.

2.12 We have found that the affect of internal degrees of freedom on the thermal conductivity coefficient of molecular systems is dependent on the density, contrary to usual assumptions.

2.13 In a nonequilibrium molecular dynamics (NEMD) study of the shear-induced melting transition in a model two-dimensional solid, we have identified two distinct regimes. At low shear rates, there is an elastic-to-plastic transition, but very long-range bond-orientational correlations are retained with a pronounced sixfold anisotropy in the instantaneous static structure factor, but at higher shear rates, the system behaves like an ordinary two-dimensional liquid under shear.

2.14 We have successfully recalculated the Weissenberg effect for a model fluid of soft spheres using density dependencies (associated with normal pressure differences) which were calculated from simulation studies of the model fluid. This has enabled us to describe the Weissenberg 
effect solely using computer simulation results for the density dependencies of all relevant quantities; the connection to rheological behavior in real fluids can now be made more convincing.

2.15 We have implemented a modified weighted density approximation algorithm together with an accurate fluid Helmholtz energy surface and direct correlation functions from realistic model potentials; in the determination of the freezing line, we have found the model to be extremely sensitive to the choice of energy surface and interatomic potential.

2.16 The computer simulations show the strong connection between the gelation mechanism and the phenomena of spinodal decomposition and nucleation in a simple fluid.

2.17 We have examined higher order derivatives in an extended corresponding states model and have shown how they can contribute to the calculated results for derived thermophysical properties.

2.18 We have investigated the dynamic scaling behavior of the structure factor of quenched systems, and have explored the fractal nature of the phenomena.

2.19 We have carefully studied the distribution functions of monomer and dimer species interacting with realistic potentials, and have calculated the moments to determine the relative average particle speeds.

2.20 In a study of the quantum collisional transfer contributions to fluid viscosity, we have shown that the classical limit agrees with our purely classical calculations; this result should help resolve any questions pertaining to the classical Rainwater-Friend theory of the first density correction to transport coefficients.

2.21 We have developed computer code to describe the phase equilibrium of Type II fluid mixtures according to the nonclassical, scaling precepts; the model includes vapor-liquid equilibrium, liquid-liquid equilibrium, and the three-phase locus; this model has been optimized for the phase equilibria of $\mathrm{CO} 2+$ methane which exhibits incipient Type II behavior.

2.22 In a study of the vapor-liquid equilibrium of azeotropic mixtures in the extended critical region, we have analyzed data for 126 dew-bubble curves for 20 mixtures using a transformed thermodynamic space which allows investigation of the details of the surface. In general, the experimental curves are wider that the theoretical ones, suggesting a need to modify the initially linear critical-locus relation between composition and the field variable $\zeta$. It may be difficult to distinguish between intrinsic binary behavior and effects of impurities.

2.23 We were able to find an extension of the Clausius-Clapeyron equation which enabled us to calculate enthalpy or entropy differences across mixture dew-bubble phase boundaries and successfully tested this method with binary fluid data in the extended critical region.

2.24 The modified Leung-Griffiths model was further extended to multicomponent mixtures of four or more components and has yielded fair agreement with data for ethane + propane $+n$-butane $+n$-pentane $+n$-hexane and to the 4-component mixtures without ethane and without $n$-hexane. A 
label-invariant measure of the distance between experimental and calculated compositions has been developed and tested for ternary mixtures.

2.25 The mode coupling results for critical thermal conductivity enhancement have been incorporated into a general extended corresponding states model and are being used as an option in our alternative refrigerants database.

2.26 We have examined critical lines of Type 3 mixtures using a full implementation of our extended corresponding states model; although we obtain qualitatively correct results for both liquidliquid and gas-gas equilibrium, the shape of the gas-gas locus for dilute nitrogen in water needs improvement.

2.27 We have examined the behavior of the "hidden" field variable, $\zeta$, (in the modified Leung-Griffiths model for mixtures in the extended critical region) and have shown how it can be made linear in the composition along the critical line for certain systems.

2.28 We have examined the asymptotic behavior of scaled critical region models and developed a procedure to extract the critical exponent $\beta$ directly from isothermal VLE data for certain dilute systems, in order to test a controversial hypothesis concerning the universality class of water-salt mixtures; our results tend to rule out the possibility of a classical critical exponent. Further study of water-salt with a crossover model confirms that the critical exponents are nonclassical.

2.29 We have used the modified Leung-Griffiths model and the Deiters equation of state to implement a critical-region thermodynamic consistency test proposed by Prausnitz. In collaboration our experimental group, we have successfully tested the method on recently measured data for ethane + propane.

2.30 We have developed techniques to integrate renormalization-group-related crossover equations into both the Leung-Griffiths model and other equation of state models.

\subsection{Research Progress}

In this section, we report on progress in each of the three major areas of the contract as defined in Section 1.0. This discussion is in narrative form to complement the very brief highlights of Section 2.0. Because a complete report on the relevant research would entail discussion of each of the publications and talks listed in Section 4.0, we restrict this section to selected topics only.

\subsection{Scattering Studies}

Statistical mechanics can show us how to calculate thermophysical properties and understand macroscopic phenomena if we know the microscopic structure of the fluid. Computer simulation of model systems, theoretically based expansions for simple potentials, or inversion of macroscopic properties can aid in our understanding of the microstructure. However, especially for complex systems near a phase boundary, direct experimental evidence is needed to complement these results. Many of the scattering experiments for this work were performed at the Cold Neutron Research 
Facility at the National Institute of Standards and Technology in Gaithersburg, Maryland. Neutron beams at the Los Alamos Neutron Scattering Center and the Institute Laue Langevin in Grenoble have also been utilized.

Various systems both in equilibrium and with an applied shear have been studied. A key element of these experiments has been the technique of contrast variation using solvents of mixed $\mathrm{H}_{2} \mathrm{O}$ and $\mathrm{D}_{2} \mathrm{O}$ to study the various contributions to the full mixture structure factor of colloidal systems, and isotopic substitution of D with $\mathrm{H}$ and ${ }^{13} \mathrm{C}$ with ${ }^{12} \mathrm{C}$ to study molecular fluids. The intensity of scattered neutrons in the momentum transfer range of interest is strongly dependent on the nuclei of these species.

Although we have discussed some results for the experiments on binary suspensions of polystyrene and silica in previous reports, we will concuntrate on the new results for this system in this section. The diameters of the latex polystyrene and silica particles are $91 \mathrm{~nm}$ and $64 \mathrm{~nm}$, giving a size ratio of about 0.7 . The volume fraction in the solution was fixed at $\varphi_{\mathrm{T}}=0.1$, and number ratios of $2,1,0.5$, and 0.25 were investigated. The wave vector range for this study was 0.025 to $0.4 \mathrm{~nm}^{-1}$. The scattering length density of polystyrene is $1.4 \times 10^{10} \mathrm{~cm}^{-2}$, that of silica is $3.6 \times 10^{10} \mathrm{~cm}^{-2}, \mathrm{H}_{2} \mathrm{O}$ is $-0.56 \times 10^{10} \mathrm{~cm}^{-2}$, and $\mathrm{D}_{2} \mathrm{O}$ is $6.4 \times 10^{10} \mathrm{~cm}^{-2}$. Thus, a solvent of $28 \% \mathrm{D}_{2} \mathrm{O}$ can match out the polystyrene in the mixture and $60 \% \mathrm{D}_{2} \mathrm{O}$ can match out the silica. The phenomenon of contrast matching implies, using an optical analogy, that the index of refraction (for neutrons) of the solvent equals that of the species in solution-thus, the particles appear invisible to the neutron beam. In this way, the experiment can examine the partial structure factors for each species in the solution as seen in Fig. 1. The data in this figure are corrected for background effects, but not for multiple scattering or polydispersity. In Fig. 2, we see directly a mixture effect on the partial structure factors by comparing results for the pure species with that of the species in the mixture.

The scattering results for the polystyrene have been analyzed with respect to density functional calculations which indicate a disordered fcc phase for these conditions. Our scattering results are not inconsistent with this analysis, although the effects of multiple scattering must be further studied. In a separate study with pure polystyrene, we have shown how the fluid structure can be varied by changing the shear rate in the system. The scattering patterns for this system are shown in Fig. 3, where the crystal symmetry is seen to depend on applied shear.

The scattering studies have proven invaluable in our general program to study properties and phenomena in fluid systems. It is necessary to have direct experimental evidence for the variation of the fluid microstructure, especially in mixtures, as the fluid approaches the solidification phase boundary. Knowledge of the degree of disorder in a polycrystalline phase, and the development and destruction of structure when gradients are present, are vital to the development of robust approaches to predict and understand these phenomena. In this context, we must study different particle morphologies, changes along the approach to a phase boundary, model suspensions as well as molecular systems, and composition as well as size ratio dependencies. Many such studies have been completed as part of this contract. 


\subsection{Molecular Dynamics, Theory of the Fluid-Solid Transition, and Kinetic Processes}

Computer experiments have become an indispensable tool in understanding fluid properties as technique and hardware have evolved. Again, the coupling between the primary experimental scattering work, basic theoretical precepts, and our understanding of macroscopic phenomena drives our research in these areas. Our first focus in this section of the report, is an important thermostatting problem which arises in nonequilibrium molecular dynamics (NEMD). This work was done in collaboration with two guest workers who visited NIST: Prof. D. Evans of the Australian National University and Dr. W. Loose of the Technical University of Berlin.

Whether one works with real experimental apparatus or with NEMD simulations, it is clear that the imposition of a shear gradient can add energy to a closed system which can raise the thermodynamic temperature of the fluid under investigation. In simulations of isothermal planar Couette flow, and in NEMD in general, several conventional thermostats have been introduced to ensure that the temperature remains constant. At low Reynolds numbers, Re, the usual kinetic temperature is appropriate: $\mathrm{T}=\Sigma_{\mathrm{i}}\left(\mathrm{p}_{\mathrm{i}}\right)^{2} /\left(3 \mathrm{mNk} \mathrm{B}_{\mathrm{B}}\right)$ where $\mathrm{p}_{\mathrm{i}}$ is the peculiar momentum of particle $i$ of the simulation, $\mathrm{m}$ is the mass, $\mathrm{N}$ is the number of particles, and $\mathrm{k}_{\mathrm{B}}$ is the Boltzmann constant. The peculiar momentum is the usual total momentum reduced by the contribution of the streaming velocity, which is stable in the laboratory coordinate system for small Re.

For large Re, it is not appropriate to assume that the local streaming velocity maintains its steady-state value of $\mathbf{u}_{s}(\mathbf{r})=\mathbf{n}_{\mathrm{x}} \curlyvee \mathrm{y}$ where $\gamma$ is the shear gradient in the $\mathrm{y}$-direction with flow in the $\mathrm{x}$ direction. Instead, we examine a Fourier decomposition of $\mathbf{u}_{s}(\mathbf{r})$ and define a more general kinetic temperature as $\mathrm{T}=\Sigma_{\mathrm{i}} \mathrm{m}\left[\mathrm{p}_{\mathrm{i}} / \mathrm{m}-\mathbf{u}_{\mathrm{s}}\left(\mathrm{r}_{\mathrm{i}}\right)\right]^{2} /\left(3\left[\mathrm{~N}-\mathrm{N}_{\mathrm{k}}\right] \mathrm{k}_{\mathrm{B}}\right)$ where the subscript $\mathrm{k}$ indicates the number of wave vectors used to determine the local streaming velocity. With this approach, instead of using a profile-biased thermostat (PBT) which imposes a linear velocity profile in the sheared system and may lead to anomalous "string" phases in the system, we introduce a profile-unbiased thermostat (PUT). A $3 \times 3$ PUT allows maximum independence of the thermostat algorithm from the imposed external shear. In Fig. 4, we show comparisons between results obtained for a Weeks-Chandler-Anderson fluid using the PBT and a $3 \times 3$ PUT thermostat.

Our NEMD study of the shear-induced melting of two-dimensional solids made use of the investigation of thermostatting algorithms. We examined a soft-disk solid at a reduced temperature of 1 and a reduced density of 1.03 and observed a transition from elastic to plastic behavior when a shear is imposed. In addition, there were qualitatively different melting mechanisms associated with both structural and dynamic changes which separate the shear-rate axis into two regimes. For imposed shear rates less than $\gamma=0.07$, the system possesses very long-range bond-orientational correlations. In this regime, the instantaneous static structure factor exhibits clear sixfold anisotropy with a pattern that rotates uniformly in time in response to the applied shear. For higher shear rates, the systems acts like an ordinary sheared two-dimensional liquid--the ranges of translational and bond-orientational correlations are comparable, and the instantaneous static structure factor no longer exhibits sixfold anisotropy. The competing possibilities of a two-stage melting scenario or a single first-order melting transition cannot be unambiguously clarified. 
As a final example in this section, we briefly describe our work on the density functional approach to locate the fluid-solid boundary using only the structural and thermodynamic properties of the homogeneous system. The relationship between these approximations to describe the grand canonical ensemble of the inhomogeneous system and the scattering and simulation studies which can explicitly examine structure in both phases is quite direct. However, to make the calculational approach tractable, we have emphasized studies on a simple monatomic system. The system of interest is argon at its freezing transition using the modified weighted density approximation (MWDA) of Denton and Ashcroft (1989). In our study, we used the full fluid thermodynamic surface obtained from the NIST standard reference database and direct correlation functions for the fluid phase based on realistic model potentials. Previous studies have shown the MWDA to work extremely well for hard-sphere systems.

A key relationship in the MWDA formalism is

$$
\hat{\rho}\left(\rho_{s}, \alpha\right)=\rho_{s}\left[1-\frac{1}{2 \beta f_{o^{\prime}}(\hat{\rho})} \sum_{G \neq 0} \exp \left[-G^{2} / 2 \alpha\right] c_{o}(G ; \hat{\rho})\right]
$$

where $\rho_{s}$ is the density in the solid phase, is the equivalent weighted density in the fluid, and $f_{0}$ and $c_{0}$ are the Helmholtz energy and direct correlation function in the fluid phase. The summation is over reciprocal lattice vectors in the postulated fcc structure of the crystalline phase. The formalism is easily implemented for hard sphere fluids, however, the resulting freezing transition cannot be easily seen to correspond to the temperature dependence of the melting line in argon.

In Fig. 5 we compare the direct correlation functions (DCF) for the hard-sphere system and the more realistic Lennard-Jones fluid. This small change in the direct correlation function is enough to suppress some roots in the MWDA equation given above. We re-scaled the weighting function and the equivalent particle number approximation, and made additional assumptions about both the density profile in the solid phase and the ideal gas contribution to the free energies. This enabled us to stabilize the solid phase and obtain a phase transition from fluid to solid. Because the equation of state now realistically describes the argon fluid, the location of the phase boundary in both temperature and pressure is quite good. Figure 6 shows the total free energies of both the solid and fluid phases and the common tangent construction which defines the transition at a reduced temperature of 1.35 . The technique is not sufficiently well developed to calculate the transition over the entire temperature range of interest, so that other approaches will be necessary.

\subsection{Properties Near the Critical Locus}

Our understanding of the properties of fluids in the vicinity of a critical locus has improved enormously in the last two decades. Unfortunately, this knowledge has not been successfully incorporated in many areas of application, and classical results have been insufficient to adequately describe certain phenomena and technologies. The goal of applying the fundamental results on static and dynamic critical behavior to important fluids, especially mixtures, is by no means complete. In this section, we concentrate on our important objective to develop a nonclassical model for Type $I$ mixtures, with both vapor-liquid equilibrium (VLE) and liquid-liquid equilibrium (LLE). 
This ambitious goal has been achieved in principle: code has been developed that explicitly calculates the phase boundaries and 3-phase locus with such a model. The starting point is our modified Leung-Griffiths model, which has provided successful fits for more than 70 Type I mixtures that display only VLE. To that reference model we have added a new Schofield construction to represent LLE, with special care to ensure that the addition does not lead to spurious and artificial phase changes such as "vapor-vapor equilibria." The model is being optimized to describe the carbon dioxide + methane system, our choice of an initial mixture to study

Although carbon dioxide + methane does not directly exhibit LLE, there is ample evidence that this system would, with a lowering of temperature, undergo liquid-liquid demixing except that it freezes first. The presence of LLE in the fluid solution to the thermodynamics of this mixture distorts the VLE surface, and explains why this mixture has been a conspicuous exception to the pattern of otherwise excellent VLE fits with the modified Leung-Griffiths model. In Fig. 7, we show a schematic phase diagram for this system, including our conjecture for the LLE and 3-phase loci. We have found clear indications of LLE behavior, with an upper critical end point (UCEP) at about $183.5 \mathrm{~K}$, in calculations using our extended corresponding states (ECS) model. The LLE curve shown in Fig. 8 is based on these ECS calculations. The UCEP is at a pressure of about $2.8 \mathrm{MPa}$ for a nearly equimolar composition $(\mathrm{x}=0.5)$.

Preliminary studies showed that the LLE construct by itself would not significantly alter the widths of the dew-bubble isotherms near critical conditions; these widths depend on the slope of loci of constant field variable $\zeta$ and derivatives of the plait point locus. Accordingly, the relation between $\zeta$ and $x$ on the critical locus, initially linear, was altered to obtain correct widths. Figure 8 shows VLE data for the carbon dioxide + methane system. The curves in Fig. 8 were generated from a Leung-Griffiths model for Type I systems with this alteration; this description represents a significant improvement over an earlier correlation. The experimental dew-bubble curves on the right side of the diagram terminate from below at the freezing locus. Note that the experimental bubble curve at $210.15 \mathrm{~K}$ (open upright triangles) has a point of inflection which cannot be replicated with the VLE model alone. With lowering of temperature and suppression of the freezing transition, such isothermal bubble curves would eventually show a horizontal point of inflection that corresponds to the UCEP. Below that point, discontinuous intervals in composition represent tie lines for the two coexisting liquids. Because the model is expressed in terms of special field variables, it is not possible a priori to determine where in measurable thermodynamic space the LLE will occur; this has been done by an iterative process.

Because the reference VLE model has already been constrained to fit the vapor pressure curves and VLE critical locus (plait point locus), an analytic function was added to the LLE Schofield construct so that the sum vanishes along these curves. Figure 9 shows our Type II model, with the LLE construct added to the reference model for carbon dioxide + methane. The two lowest isothermal bubble curves show a discontinuity in composition representing LLE, while the dew curves are continuous but with discontiuous slope as required by thermdynamic arguments. We have developed the model so that we can accurately describe the mixture data including the location of the LLE phase transition. Note that the supercritical isotherms exhibit points of inflection, as required to obtain agreement with the experimental isotherm at $210.5 \mathrm{~K}$ on Fig. 8. The correlation for carbon dioxide + methane has therefore been completed. We will further test the model on a mixture with experimentally determined LLE data in proximity to the VLE surface. Carbon dioxide + carbon disulfide is the probable candidate system. 
We have also explored ways in which the accurate scaling theory described by the modified Leung-Griffiths model can be used to understand calorimetric variables as well as the usual P-T-P-X data. Central to this study is the temperature-dependent function $\mathrm{K}$ in the definition of $\zeta$. For a $\mathrm{P}-\mathrm{T}-\mathrm{P}-\mathrm{x}$ correlation, knowledge of $\mathrm{K}$ is unnecessary, and $\mathrm{K}$ cannot be determined from such data alone. In principle, determination of enthalpy requires knowledge of $\mathrm{dK} / \mathrm{dT}$, and determination of entropy or free energy requires knowledge of $K$ itself. However, we have found two thermodynamic relationships that can be used to obtain some information about calorimetric functions in terms of only the standard Leung-Griffiths output. The first of these relationships is a mixture generalization of the Clausius-Clapeyron equation

$$
S_{D P}-S_{B P}=V_{D P} \frac{d P_{D P}}{d T^{x}}-V_{B P} \frac{d P^{B P}}{d T^{B P}}+\frac{d}{d T} \int_{P_{D P}}^{P_{B P}} V d P
$$

where BP and DP denote a bubble point and a dew point of a mixture of fixed composition at the same specified temperature, $\mathrm{S}$ is the molar entropy, and $\mathrm{V}$ is the molar volume; within the integral the variable is the overall molar volume along a two-phase path at fixed temperature. Since the integral in this equation is the difference in molar Gibbs free energies, the enthalpy difference can also be calculated.

We have developed code to implement this thermodynamic equality by performing the necessary numerical differentiation and integration, and to interpolate experimental enthalpy data which are typically presented on pairs of dew and bubble points at constant pressure, rather than temperature. Our study emphasized data in the general region of the critical locus using the required VLE and volume data from the modified Leung-Griffiths model. Figure 11 shows our results for one isopleth of the mixture n-pentane + benzene as compared with some data obtained by flow calorimetry. Figure 12 shows deviation plots for four isopleths of that mixture. The deviations are comparable to the probable experimental errors of this difficult experiment. We anticipate that future data from a flow calorimeter currently under constructuion in our laboratories will be analyzed using this technique.

The second relationship is a thermodynamic consistency test proposed by Prausnitz for data in the critical region; it has seldom been used because of a lack of a good model for describing the thermodynamic properties of mixtures near critical conditions:

$$
\ln K_{1}+\ln \frac{\phi_{1} P_{1}}{\phi_{1}^{s} P_{1}^{s}}+x_{2} \ln \frac{\phi_{2}}{\phi_{1}}+x_{2} \ln \frac{K_{2}}{K_{1}}=\int_{0}^{x_{2}} \ln \frac{K_{2}}{K_{1}} d x_{2}+\int_{0}^{x_{2}} \ln \frac{\phi_{2}}{\phi_{1}} d x_{2}+\int_{P_{S}}^{P} \frac{V^{L}}{R T} d^{\prime} P
$$

Here fluid 1 is the less volatile component, $\mathrm{K}_{\mathrm{i}}=\mathrm{x}_{\mathrm{i}} / \mathrm{y}_{\mathrm{i}}$ where $\mathrm{x}_{\mathrm{i}}$ and $\mathrm{y}_{\mathrm{i}}$ are liquid and vapor compositions respectively, $\varphi_{i}$ is a fugacity coefficient, and $S$ denotes a value at saturation. The integration is on an isotherm from the saturation curve of fluid 1 to the point in question along the liquid side of the phase boundary (rather than the two-phase path of Eq. (2)). The relation between the fugacity fraction ratio and the Leung-Griffiths field variable $\zeta$ is

$$
\frac{\phi_{2}}{\phi_{1}}=\frac{y_{1}}{y_{2} K}\left(\zeta^{-1}-1\right)
$$


where $\mathrm{K}$ is the temperature-dependent parameter in $\zeta$. From the usual Leung-Griffiths output, many of the terms above are straightforward to calculate directly. For the terms containing a contribution $-\mathrm{x}_{2} \ln \mathrm{K}$, the required difference is available. Although only the second term is not directly calculable, the equation can provide a value for this quantity, and hence for the chemical potential of fluid 1 at a two-phase point relative to the saturation value at the same temperature.

A study of the ethane + propane system has been conducted based on recently measured VLE data with coexisting densities obtained from our laboratories. Code has been developed to implement the necessary numerical integration for both the Leung-Griffiths model and for the Dieters equation of state, which had previously been shown to provide reasonable behavior in the critical region. Figure 11 shows comparisons between the Deiters equation and the Leung-Griffiths model for the seventh term in Eq. (3) and for the difference between the third and sixth terms, in the temperature interval $330 \mathrm{~K}$ $335 \mathrm{~K}$. Figure 12 shows results of the consistency test for the Leung-Griffiths model in this range and for the Deiters equation over a wider range. Some additional mixtures have been studied, and further analysis is in progress. It is clear that this high pressure consistency test can be used over the entire two-phase region and is more rigorous that the standard thermodynamic consistency test for such data.

Finally, we mention that our extended corresponding states (ECS) model has also been used to examine the critical line of type III mixtures. These mixtures, exemplified by water + carbon dioxide and water + nitrogen, exhibit gas-gas equilibrium as well as VLE. We have implemented the ECS model using the current IAPWS formulation for the thermodynamic surface of water as the reference fluid. The phase boundaries and critical line are found directly from the algorithm using shape factors defined by standard reference quality pure surfaces. The critical line is obtained from the standard conditions requiring the vanishing of the second and third composition derivatives of the Gibbs free energy. The model gives reasonable results for the mixture with carbon dioxide, but we have not been able to avoid a spurious behavior in the approach to the pure water critical point when nitrogen is introduced in the mixture. The curves are shown in Fig. 13. This problem has also been found with other corresponding states models. The direct incorporation of scaling law behavior in these models my provide an alternative approach. 

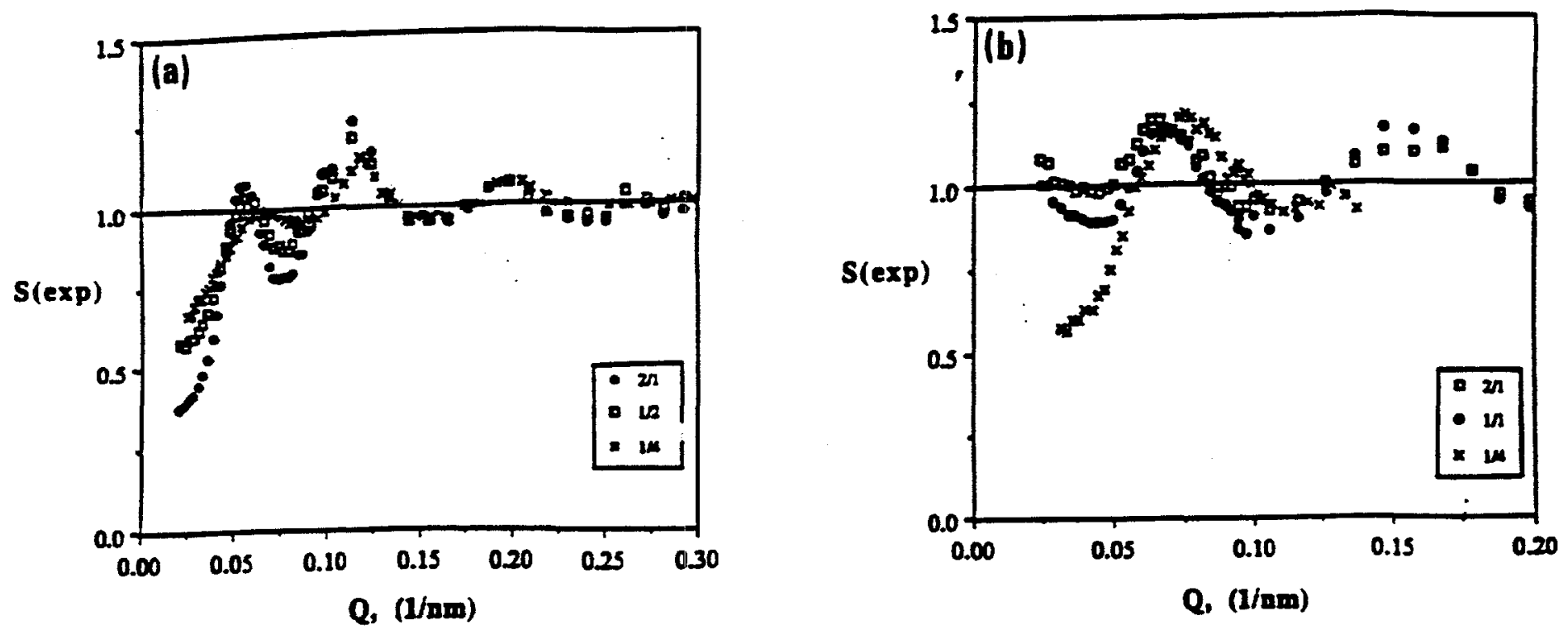

Fig. 1. Experimental partial structure factors in polystyrene - silica mixtures.
(a) polystyrene
(b) silica
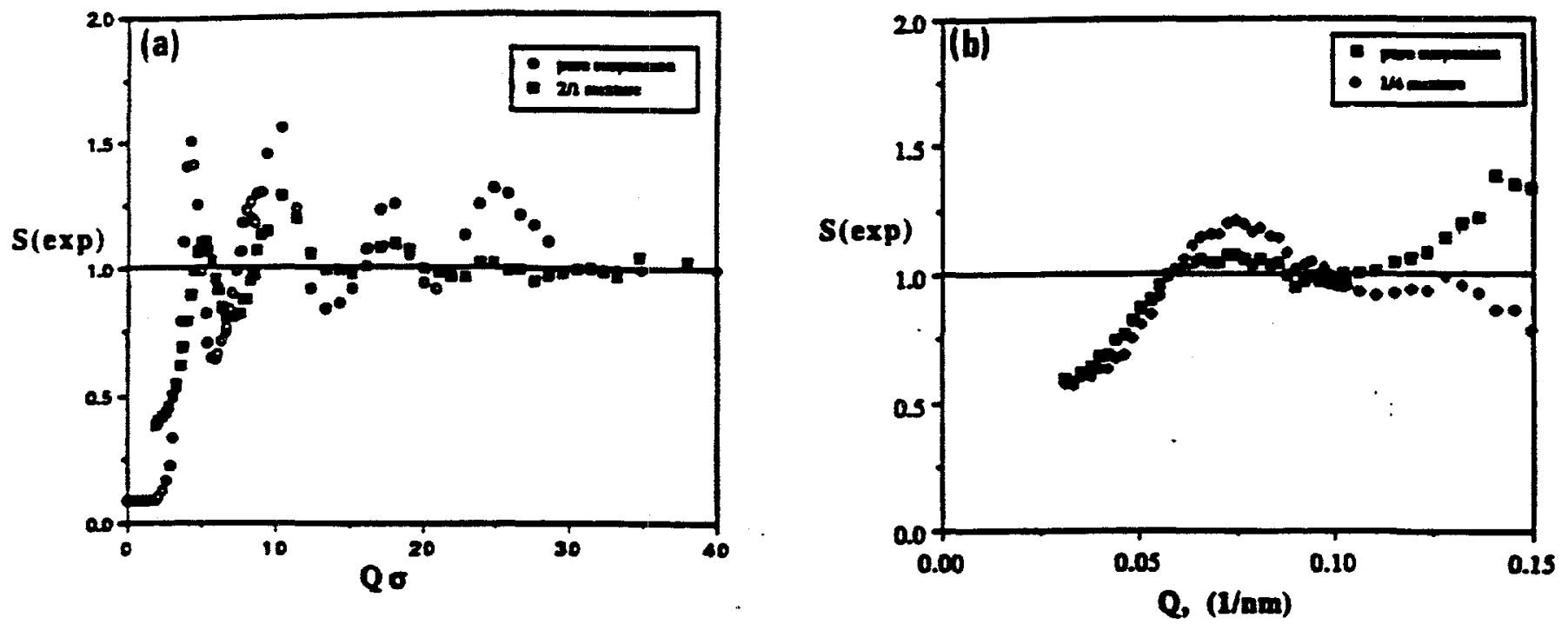

Fig. 2. Comparison between the rneasured partial structure factor and experimental structure factor for the equivalent pure suspension.
(a) polystyrene
(b) silica 


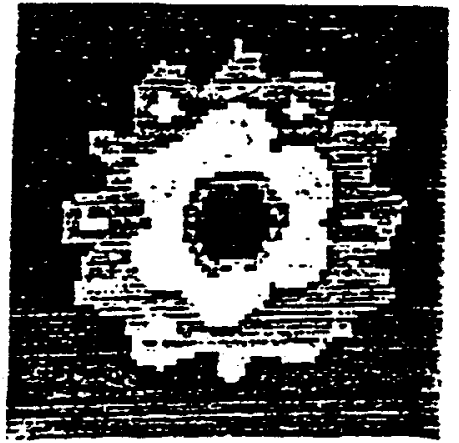

(a)

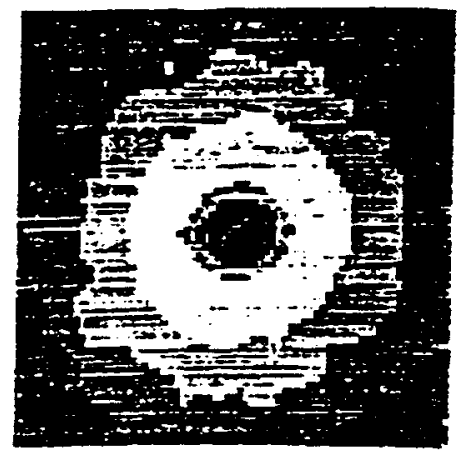

(d)

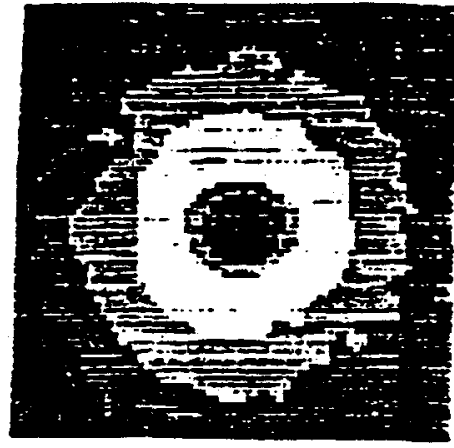

(b)

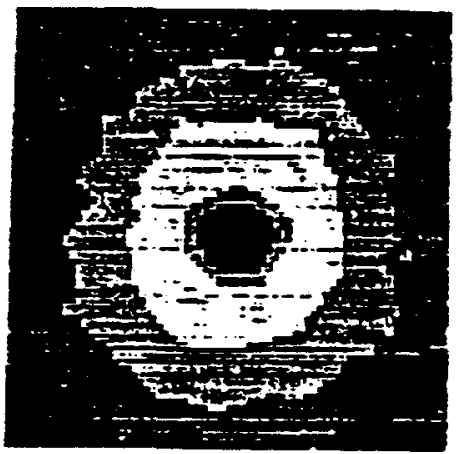

(e)

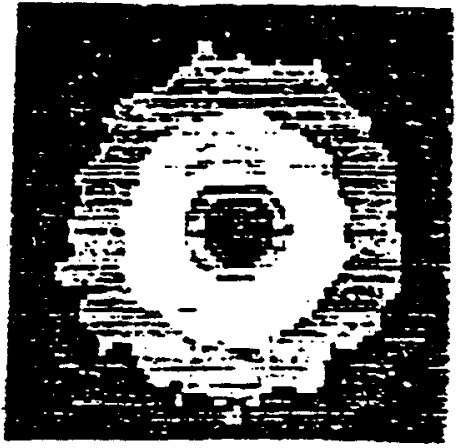

(c)

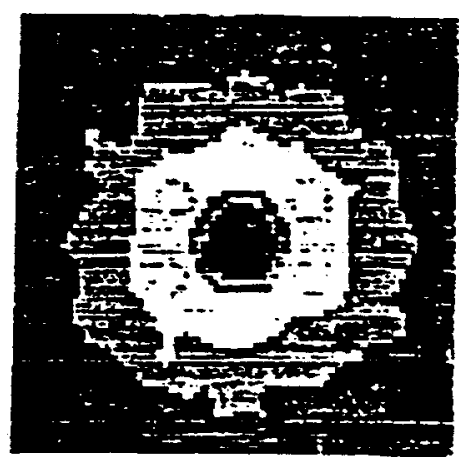

(f)

Fig. 3. Small-angle neutron scattering patterns of a monodisperse polystyrene suspension with the beam passing through the center of the shear cell perpendicular to the axis of rotation with the velocity vector pointing from left to right.

(a) no shear;

Shear rates are:
(b) $0.07 \mathrm{~s}^{-1}$
(c) $0.3 \mathrm{~s}^{-1}$
(d) $1.0 \mathrm{~s}^{-1}$
(e) $10.0 \mathrm{~s}^{-1}$
(f) $30.0 \mathrm{~s}^{-1}$ 

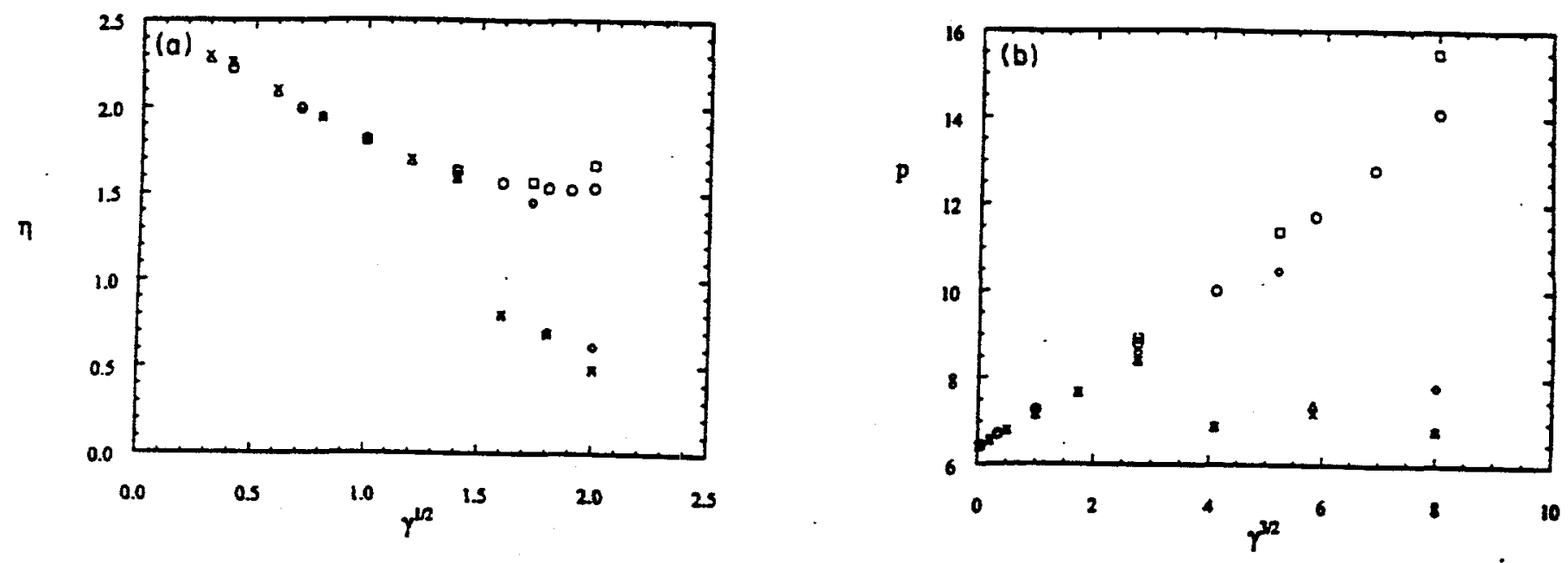

Fig. 4. (a) $\eta(\gamma)$ as a function of $\gamma 1 / 2$ for the WCA fluid using various thermostats: o denotes (3×3) PUT with $N=4000$ and $N_{k}=729$; $\square$ denotes (3×3) PUT with $N=$ 500 and $N_{k}=125 ; x$ is PBT with $N=4000$. (b) Hydrostatic pressure as a function of $\gamma 3 / 2$. 


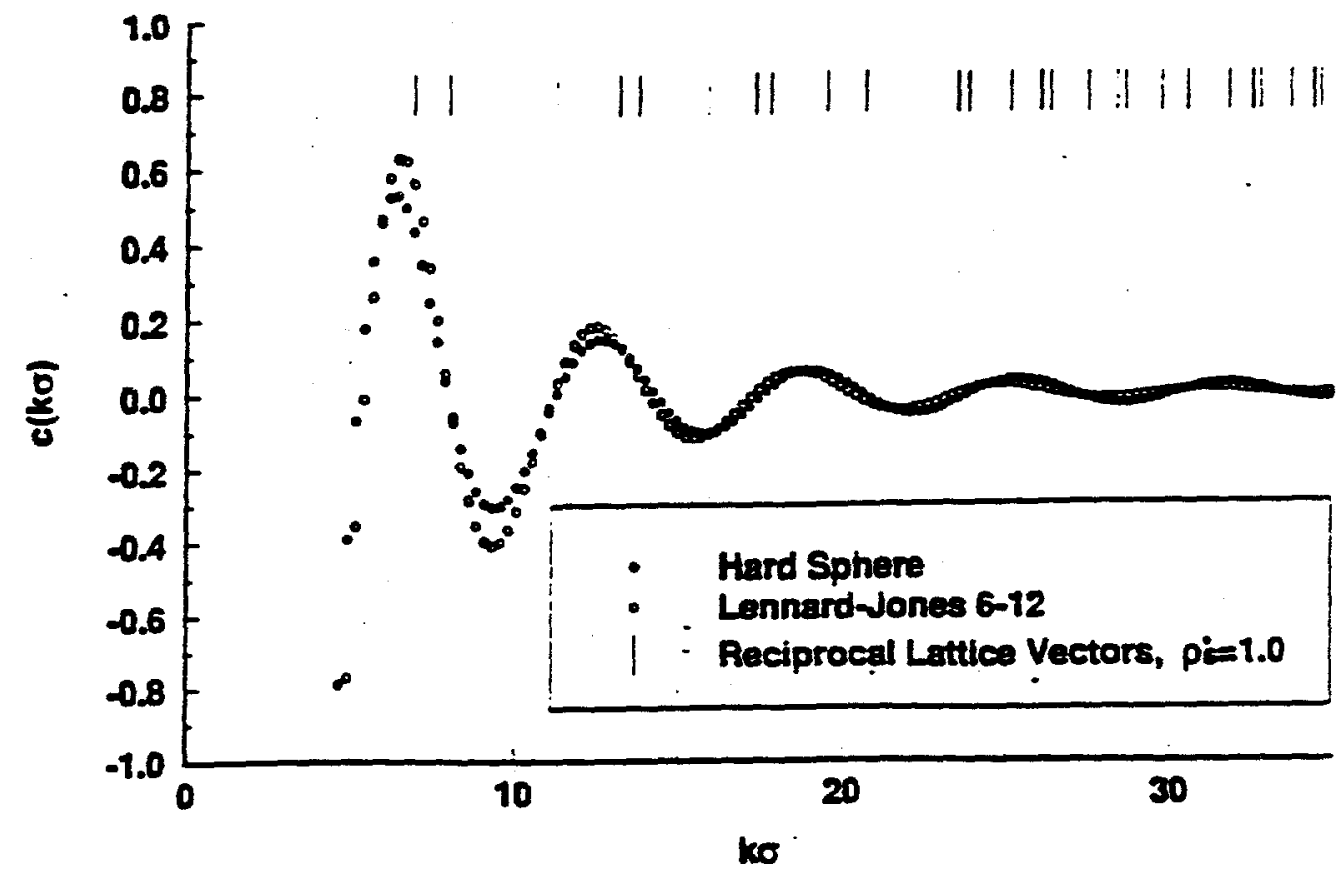

Fig. 5. Direct correlation functions for hard sphere fluid and Lennard-Jones 6-12 fluid at reduced temperature of 1.35 . Lines show position of reciprocal lattice vectors for the fcc lattice at a reduced solid density of 1 .

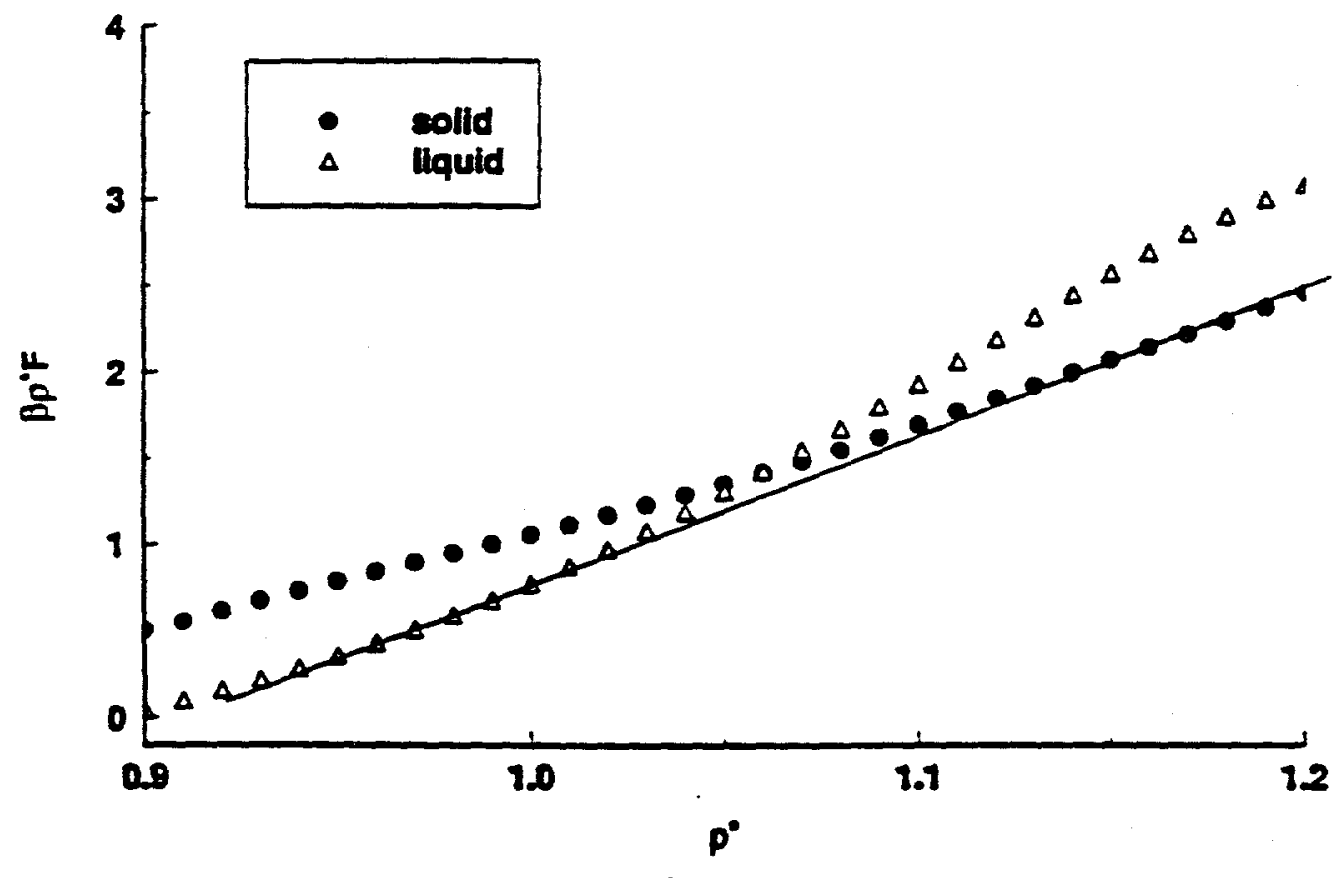

Fig. 6. Helmholtz energy of liquid and solid phases of argon at a reduced temperature of 1.35 in a modified weighted density approach. Line represents common tangent construction indicating the coexistence of the liquid phase at lower density with the solid phase at higher density. 


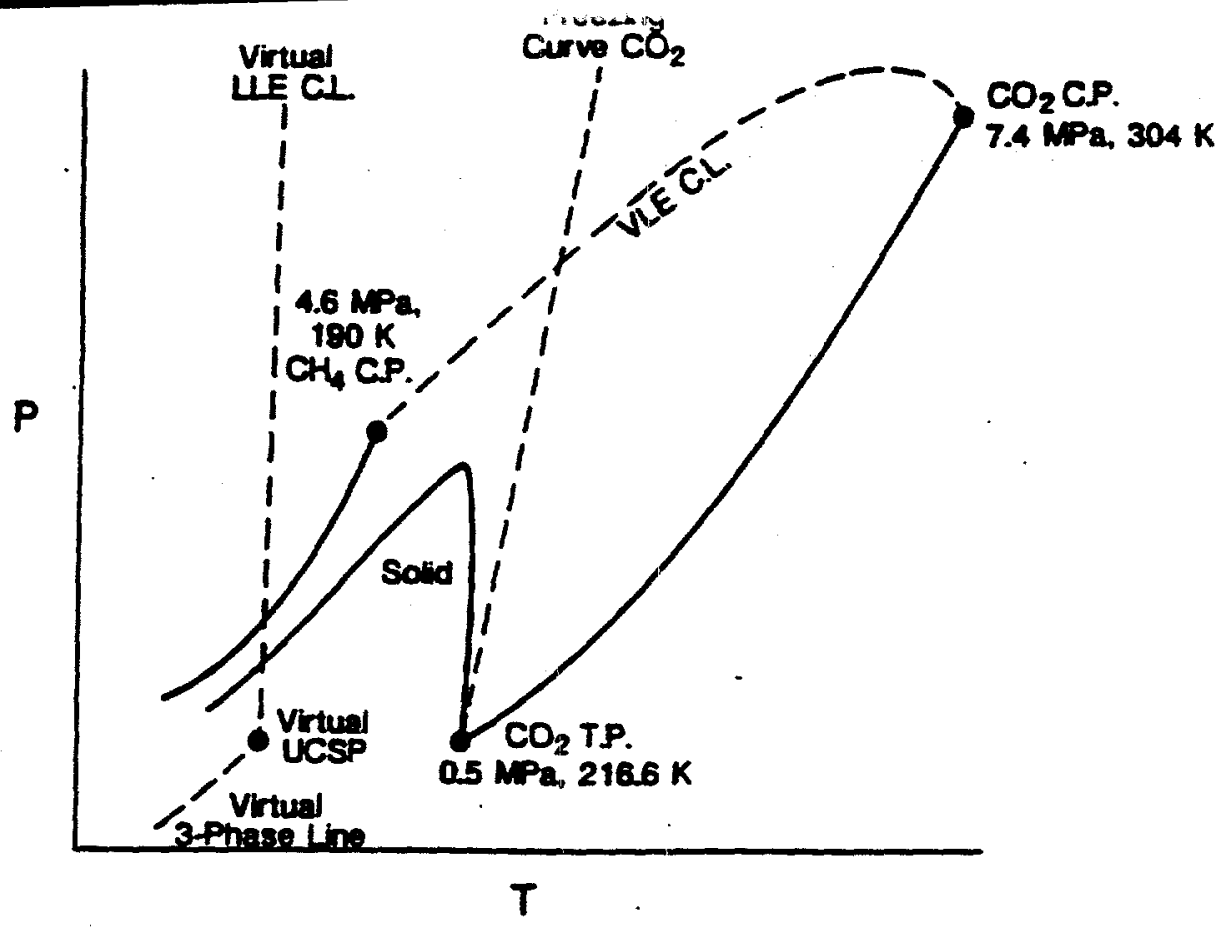

Fig. 7. Phase diagram for the methane + carbon dioxide system showing the location of the conjectured virtual LLE line.

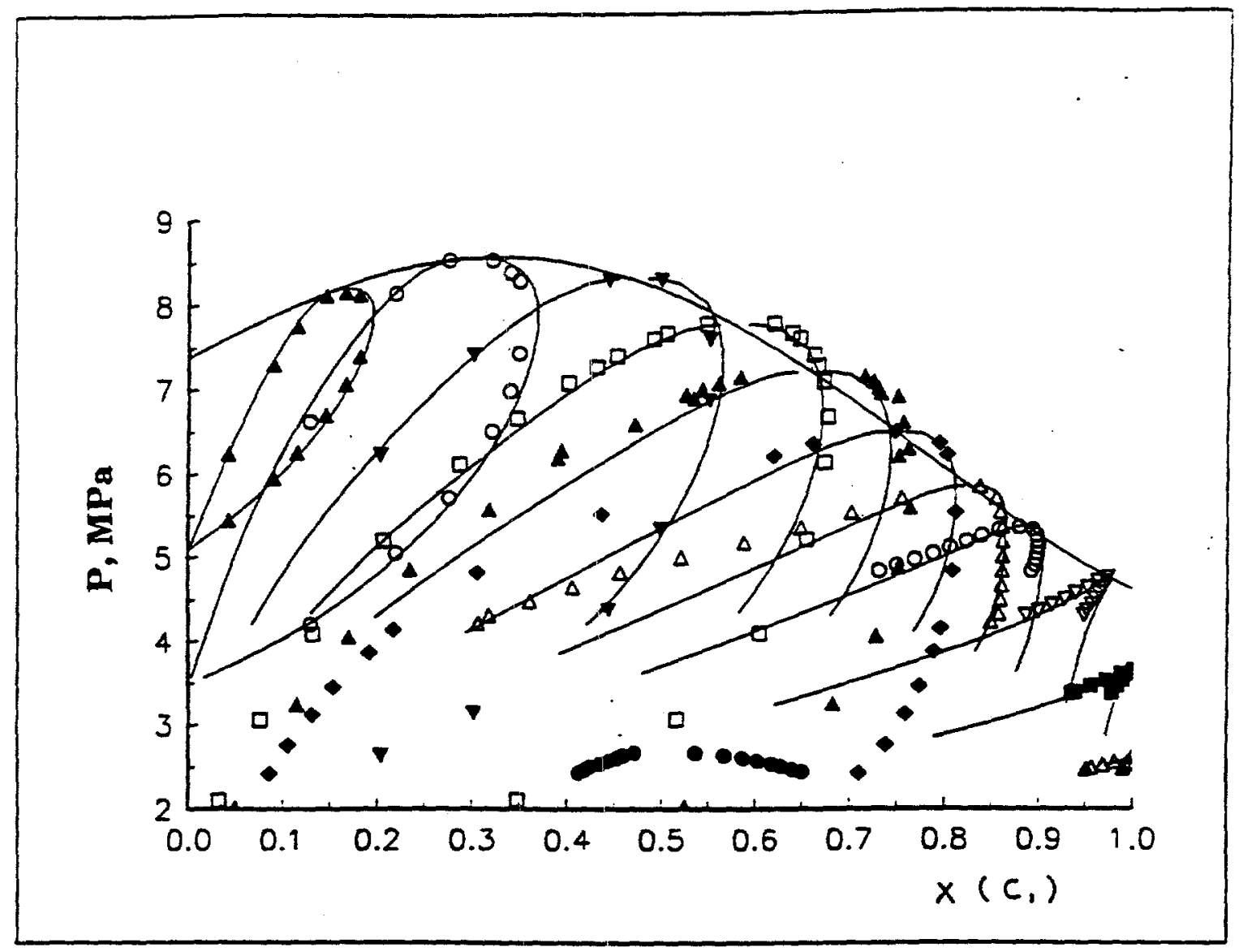

Fig. 8. Pressure-composition phase diagram of carbon dioxide + methane. Filled circles at bottom center are LLE points calculated from ECS model. Other symbols are isothermal VLE data, from $288.15 \mathrm{~K}$ at left to $173.15 \mathrm{~K}$ at right. Top solid curve is the plait point locus, and other solid curves are dew-bubble loci as calculated from a revised fit according to the modified Leung-Griffiths model. 


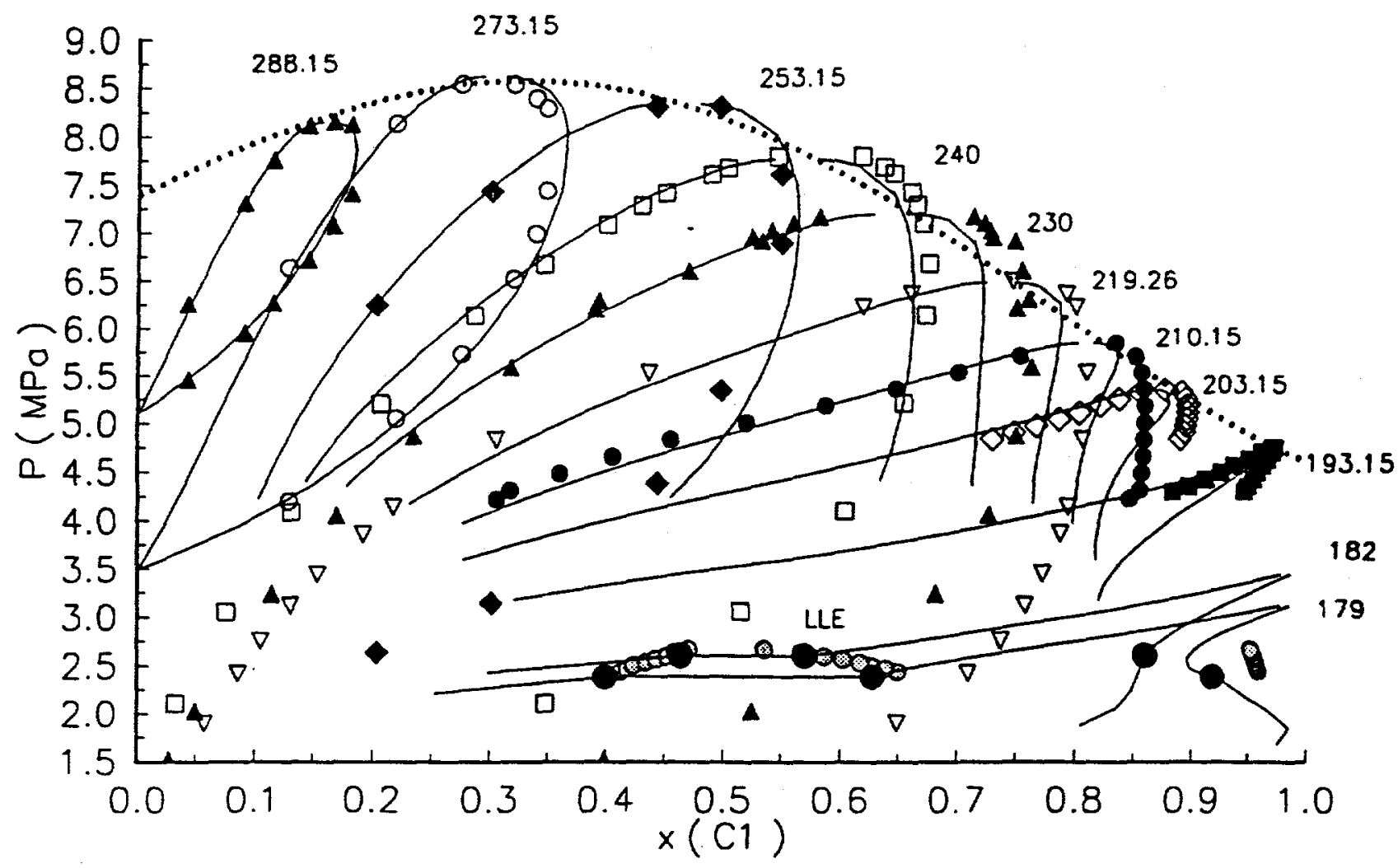

Fig. 9. Type II model, with modified Leung-Griffths fit of carbon dioxide + methane as the reference model to which is added the LLE Schofield construct. Solid lines are calculated dew and bubble curves including the lines for LIE at the two lowest temperatures. 
(a)

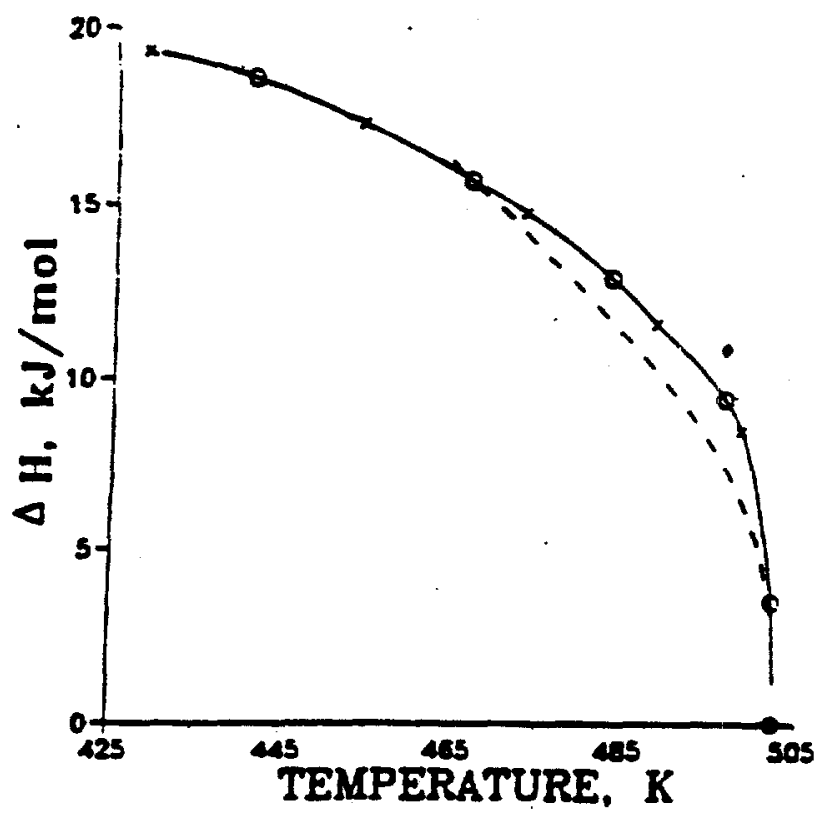

(b)

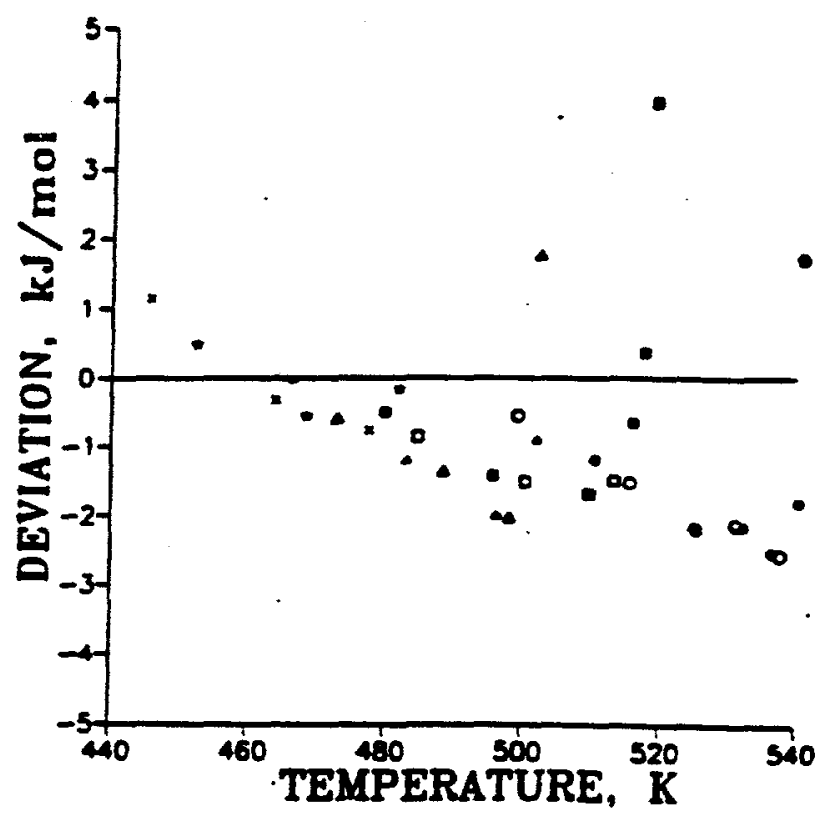

Fig. 10a. Enthalpy difference across the two-phase region for a binary muxture of $n$-pentane and benzene with mole fraction of $n$-pentane of 0.593 . Solid line is from a spline fit of the experimental data at saturated liquid temperature $(x)$ and vapor temperature (o). Dashed line is from the modified Leung-Griffiths model.

Fig. 10b. Deviations between enthalpy differences calculated from modified Leung-Griffiths model and from spline fit of enthalphy data for mixtures of n-pentane and benzene. Comparisons are made for data extending from about $1 / 2$ of the critical pressure to the critical locus. Filled symbols represent dew points and open symbols are bubble points; mole fractions of $n$-pentane range from 0.186 to 0.801 . 


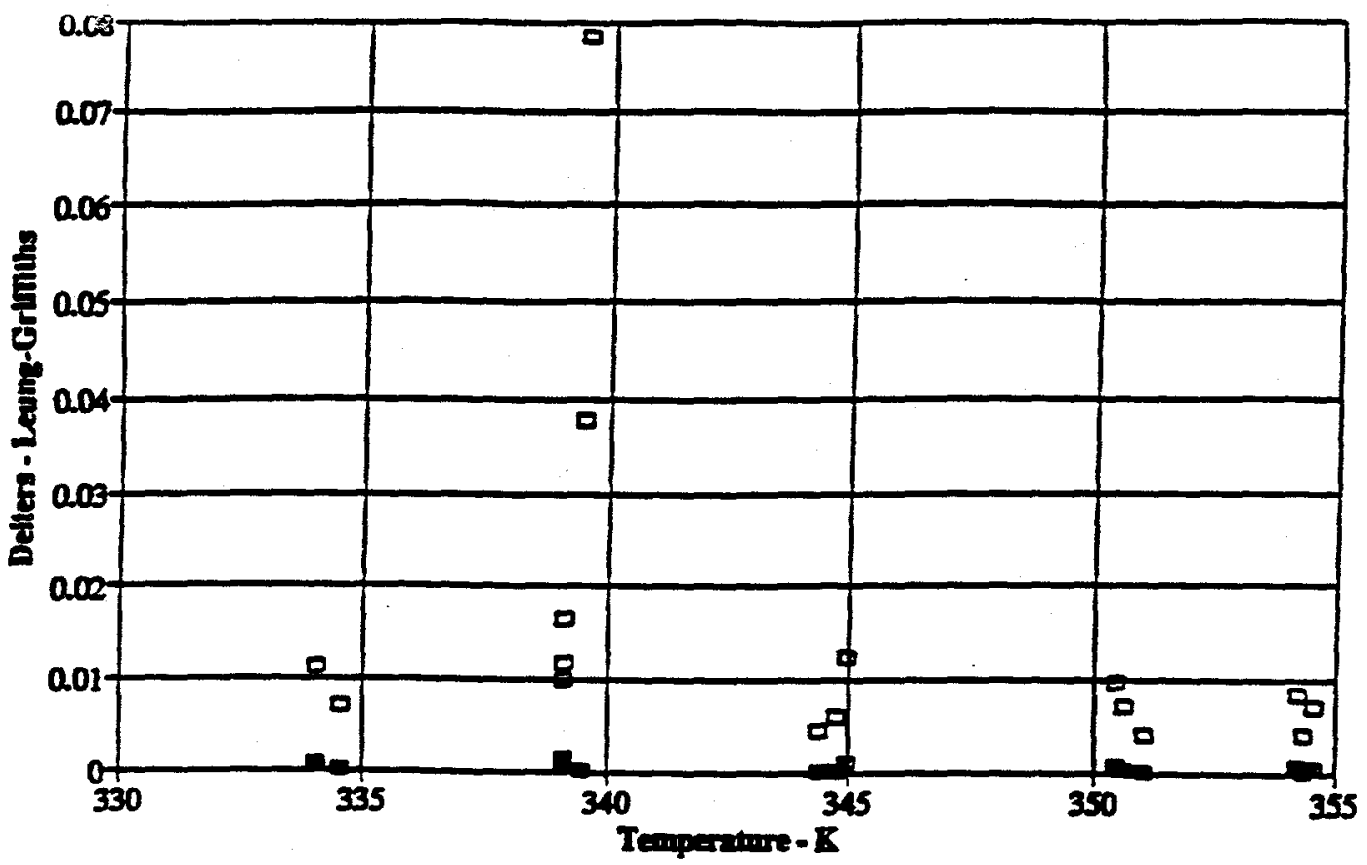

$0 \operatorname{Tegn} 3-\operatorname{Tem} 6 \operatorname{Tem} 7$

Fig. 11. Differences in the terms of Eq. (3) as calculated from the Deiters equation of state and the modified Leung-Griffiths model for the system ethane + propane.

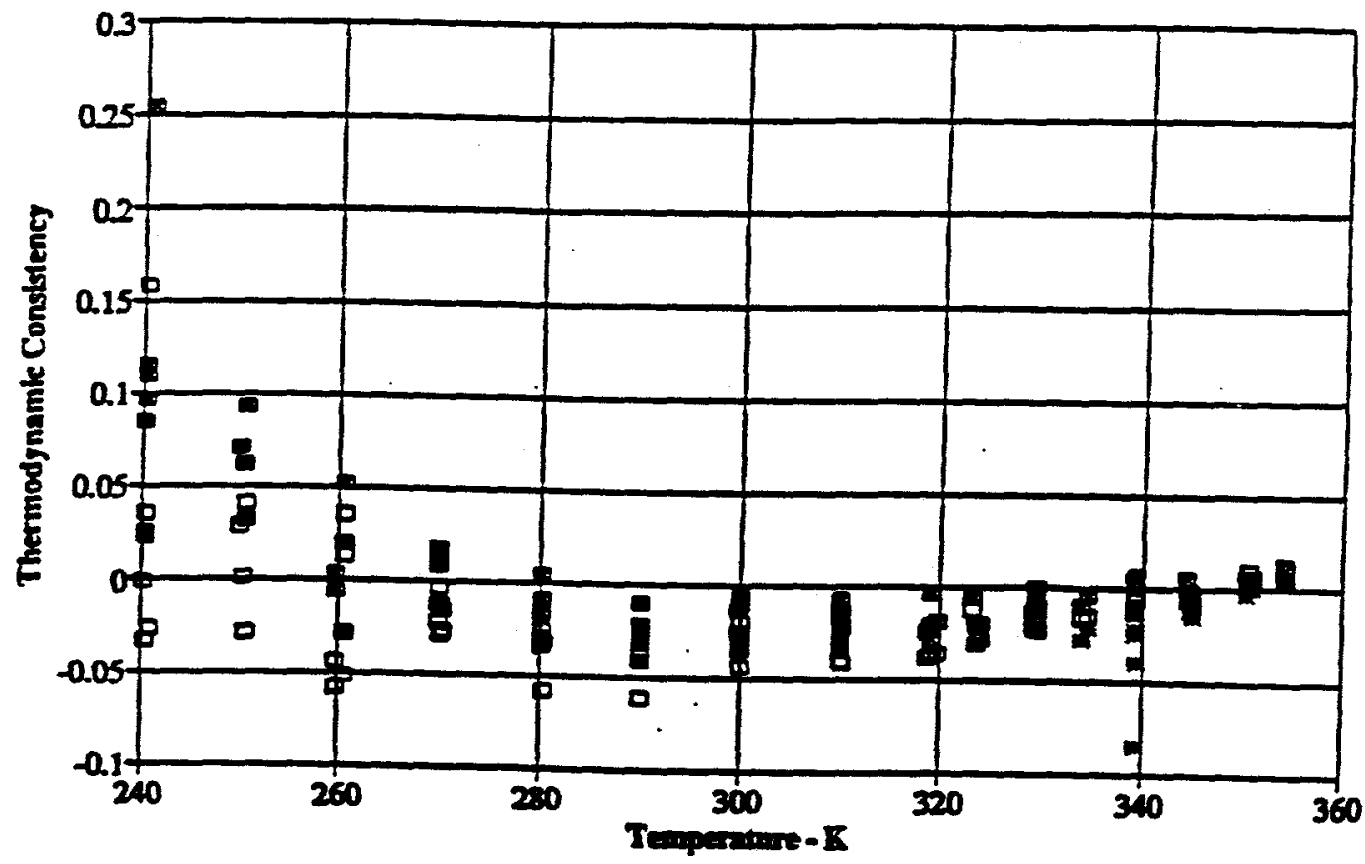

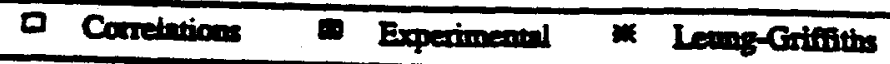

Fig. 12. Results of the critical-region consistency test from the Deiters equation and from the modified Leung-Griffiths model. 


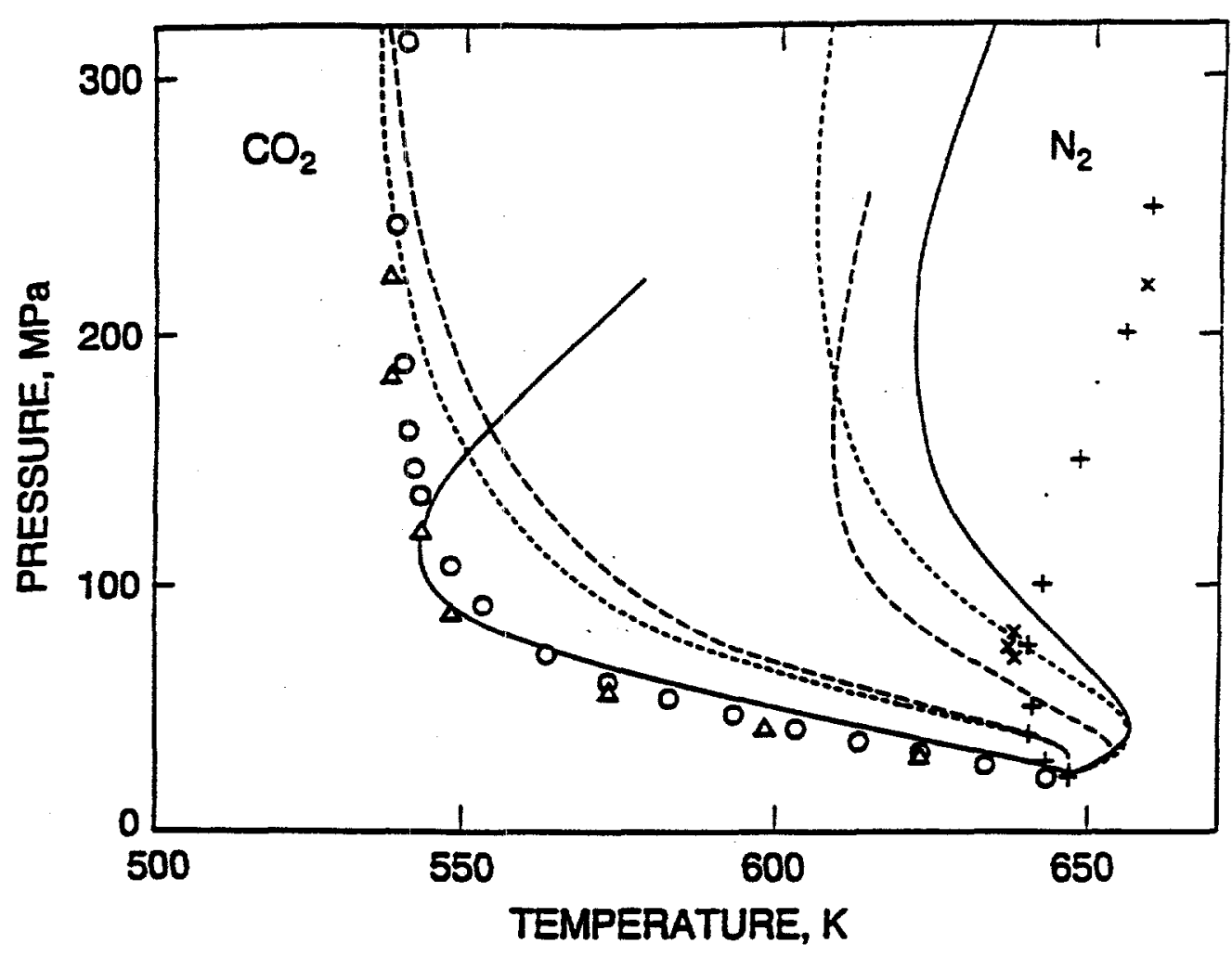

Fig. 13. Calculated curves and experimental data for critical lines of water + carbon dioxide and water + nitrogen. The dashed curves show the results of variations in a binary interaction parameter in the extended corresponding states model. 


\section{FIGURE CAPTIONS}

Fig. 1. Experimental partial structure factors in polystyrene - silica mixtures.

(a) polystyrene. (b) silica.

Fig. 2. Comparison between the measured partial structure factor and experimental structure factor for the equivalent pure suspension.
(a) polystyrene.
(b)silica.

Fig. 3. Small-angle neutron scattering patterns of a monodisperse polystyrene suspension with the beam passing through the center of the shear cell perpendicular to the axis of rotation with the velocity vector pointing from left to right.
(a) no shear;
(b) $0.07 \mathrm{~s}^{-1}$
Shear rates are.
(c) $0.3 \mathrm{~s}^{-1}$
(d) $1.0 \mathrm{~s}^{-1}$
(e) $10.0 \mathrm{~s}^{-1}$
(f) $30.0 \mathrm{~s}^{-1}$

Fig. 4. (a). $\eta(Y)$ as a function of $Y^{1 / 2}$ for the WCA fluid using various thermostats: o denotes $(3 \times 3)$ PUT with $\mathrm{N}=4000$ and $\mathrm{N}_{\mathrm{k}}=729 ; \square$ denotes (3x3) PUT with $\mathrm{N}=500$ and $\mathrm{N}_{\mathrm{k}}=125 ; \mathrm{x}$ is PBT with $\mathrm{N}$ $=4000$. (b). Hydrostatic pressure as a function of $\gamma^{3 / 2}$.

Fig. 5. Direct correlation functions for hard sphere fluid and Lennard-Jones 6-12 fluid at reduced temperature of 1.35. Lines show position of reciprocal lattice vectors for the fcc lattice at a reduced solid density of 1 .

Fig. 6. Helmholtz energy of liquid and solid phases of argon at a reduced temperature of 1.35 in a modified weighted density approach. Line represents common tangent construction indicating the coexistence of the liquid phase at lower density with the solid phase at higher density.

Fig. 7. Phase diagram for the methane + carbon dioxide system showing the location of the conjectured virtual LLE line.

Fig. 8. Pressure-composition phase diagram of carbon dioxide + methane. Filled circles at bottom center are LLE points calculated from ECS model. Other symbols are isothermal VLE data, from $288.15 \mathrm{~K}$ at left to $173.15 \mathrm{~K}$ at right. Top solid curve is the plait point locus, and other solid curves are dew-bubble loci as calculated from a revised fit according to the modified Leung-Griffiths model.

Fig. 9. Type II model, with modified Leung-Griffiths fit of carbon dioxide + methane as the reference model to which is added the LLE Schofield construct. Solid lines are calculated dew and bubble curves including the lines for LLE at the two lowest temperatures.

Fig. 10a. Enthalpy difference across the two-phase region for a binary mixture of $n$-pentane and benzene with mole fraction of $n$-pentane of 0.593 . Solid line is from a spline fit of the experimental data at saturated liquid temperature $(x)$ and vapor temperature $(0)$. Dashed line is from the modified Leung-Griffiths model. 
Fig. 10b. Deviations between enthalpy differences calculated from modified Leung-Griffiths model and from spline fit of enthalphy data for mixtures of n-pentane and benzene. Comparisons are made for data extending from about $1 / 2$ of the critical pressure to the critical locus. Filled symbols represent dew points and open symbols are bubble points; mole fractions of n-pentane range from 0.186 to 0.801 .

Fig. 11. Differences in the terms of Eq. (3) as calculated from the Deiters equation of state and the modified Leung-Griffiths model for the system ethane + propane.

Fig. 12. Results of the critical-region consistency test from the Deiters equation and from the modified Leung-Griffiths model.

Fig.13. Calculated curves and experimental data for critical lines of water + carbon dioxide and water + nitrogen. The dashed curves show the results of variations in a binary interaction parameter in the extended corresponding states model. 


\subsection{Publications, Presentations, and Graduate/Post Graduate Education}

\subsection{Publications}

Baltatu, M.E., Chong, R.A., and Huber, M.L., "Thermal Conductivity of Defined and Undefined Hydrocarbon Liquids with an Extended Corresponding States Model," in Proc. $4^{\text {th }}$ Asian Thermophysical Prop. Conf., Tokyo, Japan, 531-534 (1995).

Baltatu, M.E., Chong, R.A., and Huber, M.L., "Viscosity of Defined and Undefined Liquids Using an Extended Corresponding States Model," Int. J. Thermophysics 17, 213-221 (1996).

Butler, B.D., Hanley, H.J.M., Hansen, D. and Evans, D.J., "Aggregation of a Quenched Lennard-Jones System Under Shear," Phys. Rev. B 53, 2450-2459 (1996).

Butler, B.D., Hanley, H.J.M., Hansen, D. and Evans, D.J., "Dynamic Scaling in a 2-D Lennard-Jones System Undergoing Gas/Solid Decomposition," Phys. Rev. Lett. 74, 4468-4471 (1995).

Butler, B.D., Hanley, H.J.M., Muzny, C.D., and Straty, G.C., "Modeling of Neutron Diffraction Patterns from Sheared Silica Gels," Mat. Res. Soc. Proc. 376, 323-328 (1995).

Butler, B.D., Hanley, H.J.M., Straty, G.C., and Muzny, C.D., "Gelation Under Shear," Proc. $13^{\text {th }}$ Symp. Energy Engin. Sci., Argonne, IL, CONF-9505200, 7-15 (1995).

Butler, B.D., Hansen, D., Evans, D.J., and Hanley, H.J.M., "Aggregation of a Quenched LennardJones System under Shear," Phys. Rev. B 53, 2450 (1996).

Chen, L.B., Zukoski, C.F., Ackerson, B.J., Hanley, H.J.M and Straty, G.C., "Structural Changes and Orientational Order in a Sheared Colloidal Suspension, "Phys. Rev. Letts. 69, 688-691 (1992).

Chun, S.W., Kay, W. B. and Rainwater, J.C., "Vapor-Liquid Equilibria of Mixtures of Propane and Isomeric Hexanes," J. Chem. Eng. Data 38, 494-501 (1993).

Clarke, S.M., Rennie, A.R., Hanley, H.J.M., and Straty, G.C., "The Structure and Rheology of Hard Sphere Systems," J. Phys. Condens. Matter 6, A333 (1994).

Evans, D.J., Cui, S.T., Hanley, H.J.M and Straty G.C., "Conditions for Existence of a Reentrant Solid Phase in a Sheared Atomic Fluid," Phys. Rev. A 46, 6731-6734 (1992).

Friend, D.G. and Ely, J.F., "Thermodynamic Properties of the Methane-Ethane System," Fluid Phase Equil. 79, 77-88 (1992).

Friend, D.G. and Perkins, R.A., "Correlation Techniques," Chapter 7 in Transport Properties of Fluids: Their Correlation, Prediction, and Estimation, (J.H. Dymond, J. Millat, C.A. Nieto de Castro, Eds.) Cambridge University Press, 141-164 (1996). 
Gallagher, J.S., Friend, D.G., Given, J.A. and Levelt Sengers, J.M.H., "Critical Lines for Type-III Aqueous Mixtures by Generalized Corresponding States Models," Int. J. Thermophys. 15, 1271-1278 (1994).

Hanley, H.J.M., "Particle Dispersion in a Gas," Int. J. Thermophys. 18, 947 (1997).

Hanley, H.J.M. and Evans, D.J., "Transport Properties and Nonequilibrium Molecular Dynamics," Chapter 9 in Transport Properties of Fluids: Their Correlation, Prediction, and Estimation, (J.H. Dymond, J. Millat, C.A. Nieto de Castro, Eds.) Cambridge University Press, 210-225 (1996).

Hanley, H.J.M., Straty, G.C. and Linder, P., "Structure of a Binary Suspension of Polystyrene and Silica," Langmuir 10, $72-79$ (1994).

Hanley, H.J.M., Straty, G.C. and Tsvetkov, F., "SANS Study of a Clay Suspension under Shear," Langmuir, 10, 3362-3364 (1994).

Harvey, A.H., "Semiempirical Correlation for Henry's Constants over Large Temperature Ranges," AIChE Journal, 42, 1491-1494 (1996).

Hissong, D.W., Kay, W.B. and Rainwater, J.C., "Critical Properties and Vapor-Liquid Equilibria of the Binary System Propane-Neopentane," J. Chem. Eng. Data 38, 486-493 (1993).

Huber, M.L., "Structural Optimization of Vapor Pressure Correlations using Simulated Annealing and Threshold Accepting: Application to R134a," Comp. Chem. Eng. 18, 929-932 (1994).

Huber, M.L. and Ely, J.F., "A Predictive Extended Corresponding States Model for Pure and Mixed Refrigerants Including a New Equation of State for R134a," Int. J. Refrig. 17, 18-31 (1994).

Huber, M.L., Friend, D.G. and Ely, J.F., "Prediction of Thermal Conductivity of Refrigerants and Refrigerant Mixtures," Fluid Phase Equil. 80, 249-261 (1992).

Huber, M.L and Hanley, H.J.M., "Transport Properties from Extended Corresponding States," Chapter 12 in Transport Properties of Fluids: Their Correlation, Prediction, and Estimation, (J.H. Dymond, J. Millat, C.A. Nieto de Castro, Eds.) Cambridge University Press, 283-293 (1996).

Lin, M.Y., Hanley, Sinha, S.K. Straty, G.C., Peiffer, D.G., and Kim.W., "Shear-induced Behavior in a Solution of Cylindrical Micelles," Phys. Rev. E 53, R4302-R4305 (1996).

Lin, M.Y., Hanley, H.J.M., Straty, G.C., Peiffer, D.G., Kim, M.W. and Sinha, S.K., "Small Angle Neutron Scattering Study of Worm-like Micelles under Shear," Int. J. Thermophys. 15, 1169 (1994).

Lin, M.Y., Sinha, S.K., Hanley, H.J.M., Straty, G.C., Peiffer D.G., and Kim, M.W., "A SANS Study of Worm-like Micelles, Physica B 213 \& 214, 613-615 (1995).

Lynch, J.J. and Rainwater, J.C., "The Modified Leung-Griffiths Model of Vapor-Liquid Equilibrium: Extended Scaling and Binary Mixtures of Dissimilar Fluids," Fluid Phase Equil. 75, 23-37 (1992). 
Lynch, J.J., Rainwater, J.C., Van Poolen, L.J. and Smith, D.H., "Prediction of Fluid Phase Equilibrium of Ternary Mixtures in the Critical Region and the Modified Leung-Griffiths Theory," J. Chem. Phys. 96, 2253-2260 (1992).

Muzny, C.D., Butler, B.D., and Hanley, H.J.M., "Evolution of the Structure Factor in Gelling Dense Colloidal Silica," Mat. Res. Soc. Proc., 407, 87-92 (1996).

Muzny, C.D., Butler, B.D., and Hanley, H.J.M., "Dynamic Structure Factor Scaling in Dense Silica Gelling Suspensions, J. Phys: Condensed Matter 8, 9457-9461 (1996).

Muzny, C.D., Butler, B.D., Hanley, H.J.M., Tsvetkov, F., and Peiffer, D.G., "Silicate Clay Platelet Dispersion in a Polymer Matrix," Mat. Res. Soc. Proc., 435, 85-90 (1996).

Muzny, C.D., Butler, B.D., Hanley, H.J.M., Tsvetkov, F., and Peiffer, D.G., "Clay Platelet Dispersion in a Polymer Matrix," Mat. Lett. 28, 379-384 (1996).

Muzny, C.D., Hansen, D., Straty, G.C., Evans, D.J., and Hanley, H.J.M., "Simulation and SANS Studies of Gelation Under Shear," Int. J. Thermophys. 16, 337-346 (1995).

Muzny, C.D., Straty, G.C., and Hanley, H.J.M., "Small Angle Neutron Scattering Study of Dense Sheared Silica Gels," Phys. Rev. E 50, 675-679 (1994).

Narayan, A. P., Rainwater, J. C. and Hanley, H.J.M., "Non-Newtonian Flow Between Concentric Cylinders Using Thermophysical Properties Obtained From Simulations", Int. J. Thermophys. 16, 347353 (1995).

Ottewill, R.H., Hanley, H.J.M., Rennie, A.R., and Straty, G.C., "Small Angle Neutron Scattering Studies on Binary Mixtures of Charged Colloidal Particles," Langmuir 11, 3757-3765 (1995).

Perkins, R.A. and Friend, D.G. "Thermal Conduction in Nonmetallic Liquids," in Encyclopedia of Applied Physics, (G.L. Trigg, E.S. Vera, and W. Greulich, Eds.) VCH Publishers, New York, Vol. 21, pp. 103-132 (1997).

Rainwater, J.C., "Quantum Collisional Transfer Contributions to the Density Dependence of Gaseous Viscosity," in Proc. 19th Int. Symp. Rarefied Gas Dynamics, Oxford Univ. Press, Oxford, 114-120 (1995).

Rainwater, J.C. and Friend, D.G., "Calculation of Enthalpy and Entropy Differences of Near-Critical Binary Mixtures with the Modified Leung-Griffiths Model," J. Chem. Phys. 98, 2298-2307 (1993).

Rainwater, J.C. and Friend, D.G., "Composition Dependence of a Field Variable Along the Binary Fluid Mixture Critical Locus," Phys. Lett. A. 191, 431-437 (1994).

Rainwater, J.C., Hanley, H.J.M., and Narayan, A., "The Density Dependence of Fluid Properties and Non-Newtonian Flows: the Weissenberg Effect," J. Rheology 39, 1343-1359 (1995). 
Rainwater, J.C. and Lynch, J.J., "A Nonlinear Correlation of High-Pressure Vapor-Liquid Equilibrium Data for Ethylene + n-Butane Showing Inconsistencies in Experimental Compositions," Int. J. Thermophys. 15, 1231-1239 (1994).

Rainwater, J.C. and Snider, R.F., "The Equilibrium Pair Distribution Function of a Gas: Aspects Associated with the Presence of Bound States," J. Chem. Phys. 99, 9111-9121 (1993).

Ravi, P., Murad, S., Hanley, H.J.M. and Evans D.J., "The Thermal Conductivity of Polyatomic Molecules: Benzene," Fluid Phase Equil. 76, 249-257 (1992).

Van Poolen, L.J. and Rainwater, J.C., "Vapor-Liquid Equilibria of Ternary Mixtures in the Critical Region on Paths of Constant Temperature and Overall Composition," Int. J. Thermophys. 16, 473-481 (1995).

Weider, T., Glaser, M.A., Hanley H.J.M and Clark, N.A., "Shear-induced Melting of Two Dimensional Solids," Phys. Rev. B 47, 5622-5628 (1993).

Welberry, T.R. and Butler, B.D., "Diffuse x-ray Scattering from Disordered Crystals," Chem. Rev. 95, 2369-2403 (1995).

Welberry, T.R. and Butler, B.D., "Local Structural Information of Mullite Obtained from Diffuse Xray Scattering," J. European Ceramic Soc. 16, 187-193 (1996).

\subsection{Graduate and Post-Graduate Education}

Butler, B., 1994 - 1996, NIST/NRC Postdoctoral Research Fellow, from Northwestern University, advisor H.J.M. Hanley.

Gay, S., 1996 - NIST PREP Graduate Research Assistant, University of Colorado, Department of Physics, thesis advisor J.C. Rainwater.

Lynch, J.J., 1986 - 1990, Graduate Research Assistant, PhD 1990, University of Colorado, Department of Physics, thesis advisor J.C. Rainwater.

Lewandowska, A, 1993 - 1997, NIST PREP Graduate Research Assistant, MS 1994, University of Colorado, Department of Electrical Engineering, advisor H.J.M. Hanley.

Muzny, C.D, 1995 - 1998, NIST PREP Postdoctoral Research Assistant, from University of Colorado, Department of Physics, advisor H.J.M. Hanley.

Narayan, A.P., 1992 - 1996, NIST Graduate Research Assistant, MS 1994, University of Colorado, Department of Chemical Engineering, thesis advisor H.J.M. Hanley. 


\subsection{Presentations}

Friend, D.G. (speaker), M.L. Huber, and J.S. Gallagher, "Thermophysical Property Computer Packages from NIST," November 9, 1992, ASME Winter Annual Meeting, Anaheim, CA.

Friend, D.G. (speaker) and W.M. Haynes, "Reference Data for the Thermophysical Properties of Fluids," March 31, 1993, A.I.Ch.E. Spring National Meeting, Houston.

Friend, D.G., "Standard Reference Data in Thermodynamics and Thermophysical Properties for Aerospace Applications," April 1, 1993, SAE Committee S-15, Mtg. 28, Phoenix.

Friend, D.G. and W.M. Haynes, "Properties of Ammonia-Water Mixtures," NREL Seminar, June 22, 1993, Golden, Colorado.

Friend, D.G., "Thermophysical Properties of the Ammonia-Water System," IAPWS Working Group Report, September 20, 1993, Milan.

Friend, D.G., "Modeling the Properties of Air at Very High Temperatures and Pressures," December 6, 1993, Princeton Hypersonic Tunnel Workshop; Princeton, New Jersey.

Friend, D.G., "Standard Reference Databases for the Thermophysical Properties of Fluids," March 13, 1994, American Chemical Society 207th Nat. Mtg., San Diego, California.

Friend, D.G. and Huber, M.L. (speaker), "Thermophysical Property Standard Reference Data from NIST," June 21, 1994, 12th Symposium on Thermophysical Properties, Boulder, Colorado.

Friend, D.G., "Standard Thermophysical Properties of the Ammonia-Water Binary Fluid," September 12, 1994, 12th International Conference on the Properties of Water and Steam, Orlando, Florida.

Friend, D.G., "Calculation of Fluid Properties using Extended Corresponding States Methods," October 7, 1994, University of Nevada at Reno, Reno, Nevada.

Friend, D.G., "Fluid Property Standards," December 1, 1994, NIST, Boulder, Colorado.

Gallagher, J.S. (speaker), Friend, D.G., Given, J.A. and Levelt Sengers, J.M.H.,"Critical Lines for Type-III Mixtures by Generalized Corresponding-States Models," June 22, 1994, 12th Symposium on Thermophysical Properties, Boulder, Colorado.

Gallagher, J.S., Friend, D.G., and Levelt Sengers, J.M.H., "Thermodynamic Properties of Solutions of Air Constituents in Near and Supercritical Water," sched. September 13, 1994, 12th International Conference on the Properties of Water and Steam, Orlando, Florida.

Hanley, H.J.M., "Structures of Mixtures," February 6, 1992, Australian National University, Canberra, Australia. 
Hanley, H.J.M., "Neutron Scattering Studies," February 20, 1992, University of Western Australia, Perth, Australia.

Hanley, H.J.M., "Properties of Liquid Mixtures," February 21, 1992, Murdoch University, Perth, Australia.

Hanley, H.J.M., "Structures of Mixtures," August 4, 1992, 18 STATPHYS Meeting, Berlin, Germany.

Hanley, H.J.M., "Thermophysical Property Research at NIST, Boulder," March 16, 1993, Imperial College, London.

Hanley, H.J.M., "Structures of Liquids," April 9, 1993, Technical University, Berlin, Germany.

Hanley, H.J.M., "Spinodal Decomposition," December 6, 1993, Australian National University, Canberra, Australia.

Hanley, H.J.M., "A SANS Study of Gelation," February 10, 1994, Imperial College, London, United Kingdom.

Hanley, H.J.M., "Contrast Matching in Mixtures," March 23, 1994, University of Bristol, Bristol, England.

Hanley, H.J.M., "Gelation under Shear," April 13, 1994, Technical University, Berlin, Germany.

Hanley, H.J.M., "Fluids, Who Cares?," May 3, 1994, NIST, Boulder, Colorado.

Hanley, H.J.M., "Fluid Research in the 1990's," June 8, 1994, NIST, Gaithersburg, Maryland.

Hanley, H.J.M., "The Structure of Dense Gels," November 13, 1994, University of Colorado, Boulder, Colorado.

Hanley, H.J.M., "The Structure of Dense Gels," November 29, 1994, Technical University, Berlin, Germany.

Hanley, H.J.M., "Thermal Conductivity in the Critical Region," February 17, 1995, Australian National University, Canberra, Australia.

Hanley, H.J.M., "Sol-gel Processing," October 15, 1995, Colorado School of Mines, Golden, Colorado.

Holcomb, C. D. (speaker) and Rainwater, J. C., "Evaluation of a High Pressure Thermodynamic Consistency Test Using Ethane + Propane and Propane + n-Butane in the Critical Region," June 24, 1994, 12th Symposium on Thermophysical Properties, Boulder, Colorado.

Huber, M.L. and Friend, D.G. (speaker), "Calculation of Melting Lines using a Density Functional Theory," June 22, 1994, 12th Symposium on Thermophysical Properties, Boulder, Colorado. 
Muzny, C.D., "Evolution of the Structure Factor in Gelling Dense Colloidal Silica," November, 28, 1995, Materials Research Society Fall Mtg., Boston, Massachusetts.

Narayan, A. P., Rainwater, J. C. and Hanley, H. J. M. (speaker), "Non-Newtonian Flow Between Concentric Cylinders Using Thermophysical Properties Obtained from Simulations," June 22, 1994, 12th Symposium on Thermophysical Properties, Boulder, Colorado.

Rainwater, J.C., "A Theoretical Analysis of Double Retrograde Vaporization," November 20, 1991, AIChE Annual Meeting, Los Angeles, California.

Rainwater, J.C., "Calculation of Collision Integrals Arising in Moderately Dense Gas Kinetic Theory," Juiy 15, 1992, University of British Columbia, Vancouver, BC, Canada.

Rainwater, J.C. (speaker) and Friend, D.G., "Theory of Linear Density Corrections to Viscosity and Thermal Conductivity of Gases," July 28, 1992, 18th International Symposium on Rarefied Gas Dynamics, Vancouver, BC, Canada.

Rainwater, J.C. (speaker) and Friend, D.G., "Calculations of Entropy, Enthalpy, and Free Energy Differences of Near-Critical Mixtures with the Modified Leung-Griffiths Model," August 21, 1992, 12th IUPAC Conference on Chemical Thermodynamics, Snowbird, Utah.

Rainwater, J.C., "Liquid-Liquid and Liquid-Vapor Phase Changes in Mixtures," November 20, 1992, University of Colorado, Denver.

Rainwater, J.C. (speaker) and D.G. Friend, "Isothermal Vapor-Liquid Enthalpy Differences of Nearcritical Binary Mixtures," March 31, 1993, A.I.Ch.E. Spring National Meeting, Houston.

Rainwater, J.C., "Progress on a NonClassical Model of Mixtures with Vapor-Liquid and Liquid-Liquid Equilibrium," May 24, 1993, Midwest Thermodynamics Symposium, Houghton, Michigan.

Rainwater, J. C. (speaker) and Lynch, J. J., "A Nonlinear Correlation of High-Pressure Vapor-Liquid Equilibrium Data for Ethylene + n-Butane Showing Inconsistencies in Experimental Compositions," June 20, 1994, 12th Symposium on Thermophysical Properties, Boulder, Colorado.

Rainwater, J.C., "A Nonclassical Model of a Type 2 Mixture with Vapor-Liquid and Liquid-Liquid Equilibria Connected by a Three-Phase Locus," June 23, 1994, 12th Symposium on Thermophysical Properties, Boulder, Colorado.

Rainwater, J. C., "Quantum Collisional Transfer Contributions to the Density Dependence of Gaseous Viscosity," July 25, 1994, 19th International Symposium on Rarefied Gas Dynamics, Oxford, England.

Rainwater, J. C., "Phase Equilibria of Mixtures in the Critical Region," September 9, 1994, University of Southern Florida, Tampa. 
Rainwater, J. C., "An Asymptotic Expression for Phase Boundaries of Mixtures with a Dilute Nonvolatile Solvent: Application to Aqueous Salt Systems," September 14, 1994, 12th International Conference on the Properties of Water and Steam, Orlando, Florida.

Rainwater, J. C., "Density Corrections to Viscosity and Thermal Conductivity of Gases with Realistic Potentials," October 11, 1994, Eringen Symposium, 31st Annual Technical Meeting of the Society of Engineering Science, College Station, Texas.

Rainwater, J.C., "A Nonclassical Model of a Type 2 Mixture with Vapor-Liquid and Liquid-Liquid Equilibria Connected by a Three-Phase Locus," June 21, 1995, $7^{\text {th }}$ Internat. Conf. Fluid Properties and Phase Equil for Chem. Proc. Design, Snowmass Village, Colorado.

Rainwater, J.C., "Theory of the Viscosity of Dense Gases," December 1, 1995, Chemical Engin. Dept., University of Massachusetts, Amherst, Massachusetts.

Van Poolen, L. J. (speaker) and Rainwater, J. C., "Vapor-Liquid Equilibria of Ternary Mixtures in the Critical Region on Paths of Constant Temperature and Overall Composition," June 23, 1994, 12th Symposium on Thermophysical Properties, Boulder, Colorado. 